\title{
Highlights of LHAASO Science Results
}

\author{
Zhen $\mathrm{Cao}^{a, *}$ on behalf of the LHAASO Collaboration \\ (a complete list of authors can be found at the end of the proceedings) \\ ${ }^{a}$ Key Laboratory of Particle Astrophyics, Institute of High Energy Physics, Chinese Academy of \\ Sciences, \\ 100049 Beijing, China \\ E-mail: caozh@ihep.ac.cn
}

The Large High Altitude Air Shower Observatory (LHAASO) is a complex of extensive air shower (EAS) detector array for very-high energy and ultra-high energy $\gamma$-ray observation and cosmic ray studies. LHAASO was completed its construction and has been starting the full-duty operation in July 2021 at Mt Haizi 4,410 meters above sea level, China. With the data collected in 2020, LHAASO found a dozen UHE gamma-ray sources with high significance $(>7 \sigma)$. Their energy spectra are found extended to about $1 \mathrm{PeV}$ without obvious cut-off. LHAASO also recorded the unprecedent highest energy photon with the energy of $1.4 \pm 0.13 \mathrm{PeV}$. These findings confirmed the existence of PeV particle accelerators in the Milk Way and opened up an era of UHE gamma-ray astronomy. LHAASO measured the energy spectrum of the Crab Nebula from $0.5 \mathrm{TeV}$ up to $1.1 \mathrm{PeV}$ for the first time. LHAASO achieved the absolute energy scale calibration in cosmic ray shower measurements at $21 \pm 6 \mathrm{TeV}$ by measuring the Moon shadow drifting in the geo-magnetic field using WCDA. In this proceeding, we summarize the status of LHAASO experiment and highlight the $\gamma$-ray results and cosmic ray progresses.

\footnotetext{
*** 37th International Cosmic Ray Conference (ICRC2021), ***

*** 12-23 July 2021 ***

*** Berlin, Germany - Online ***
}

\footnotetext{
${ }^{*}$ Presenter
} 


\section{Introduction}

The origin of $\mathrm{PeV}$ Cosmic Rays (CRs) in the Galaxy is a long-standing puzzle. It is widely believed that there are sources in our galaxy can at least accelerate particles to $\mathrm{PeV}$ (PeVatrons). Identification of these accelerators is a prime objective towards the understanding of the origin of galactic CRs. Gamma rays and neutrinos, produced in the interaction of CRs with the ambient medium within or around the accelerator, are key signatures of these CR factories. The typical energy of gamma rays produced in the interaction of CRs with ambient medium is about $10 \%$ of the parent CR energy. A decisive indication of acceleration of $\mathrm{PeV}$ protons is the observations of ultra-high energy (UHE; $\mathrm{E} \geq 0.1 \mathrm{PeV}$ ) gamma-rays. Thus the detection of gamma rays above tens of $\mathrm{TeV}$ is crucial for tracing the accelerators.

On the other hand, accurate measurements of CR energy spectra, compositions and anisotropy for different compositions are also important tools to investigate the origin, the acceleration and the propagation of CRs [1]. For example, the 'knee' structure of CR spectrum might hint the highest energy that the Galactic CR sources can reach in accelerating particles, and the energy of the knees for different elemental compositions might reveal whether the acceleration mechanism is related to rigidity or not. Above several EeV, CRs are produced from extragalactic sources as indicates by the CR anisotropy detected by experiments, such as AUGER[2].

The Large High Altitude Air Shower Observation(LHAASO) experiment is a dualtask facility designed for $\mathrm{CR}$ and $\gamma$-ray studies at $\mathrm{TeV}$ and $\mathrm{PeV}$ energies. LHAASO can continuously monitor a large portion of the sky corresponding to almost $2 / 3$ of the celestial sphere for observations with a maximum zenith angle of $50^{\circ}$. With the hybrid observation and excellent $\gamma$ /background discrimination ability, LHAASO can survey $\gamma$-ray sources over almost 4 decades of energy with a sensitivity $\simeq 1.3 \%$ in Crab unit per year above $50 \mathrm{TeV}$. Observational results with partially deployed LHAASO array since 2019 are presented in this proceeding.

\section{LHAASO Experiment}

The LHAASO experiment [3] is located at Mountain Haizi, altitude of 4410m a.s.l. and $29^{\circ} 21^{\prime} 27.6^{\prime \prime}$ N, 10008'19.6" E, Daocheng, Sichuan Province, China. LHAASO consists of three main detector arrays, i.e. Kilometer-square Array (KM2A), Water Cherenkov Detector Array (WCDA), Wide Field of View Cherenkov Telescope Array (WFCTA).

The Water Cherenkov Detector Array (WCDA) is designed mainly for monitoring the northern sky for transient phenomena and surveying for new sources. WCDA is composed of 3 sub-arrays, i.e. WCDA-1 and WCDA-2 with an area of $150 \mathrm{~m} \times 150 \mathrm{~m}$ each, and WCDA-3 of $300 \mathrm{~m} \times 110 \mathrm{~m}$. WCDA has been built in 3 phases. WCDA-1 is completed in 2019 and consisting of 900 detector units, which are divide into 25 clusters. Each cluster has a junction box containing readout electronics and calibration facilities of 4 groups of 9 detector units. Each detector unit is $5 \mathrm{~m} \times 5 \mathrm{~m}$ that includes one 1.5 inch PMT and one 8 inch PMT to measure the charge and timing of water Cherenkov signals. It was in operation 
since April 2019. In the second phase, WCDA-2 with the same size was built and put in operation since March of 2020. In the third phase, WCDA-3 began operation at the March of 2021. For $\gamma$-ray induced showers, WCDA-2 and WCDA-3 have a threshold below 100 GeV, while WCDA-1 has the threshold of $500 \mathrm{GeV}$, because the 20" PMT and 3" PMT are used to replace the the original design of the combination of $8 "+1.5 "$ PMT pairs. [4]. A typical event recorded by WCDA is shown in Fig.1.
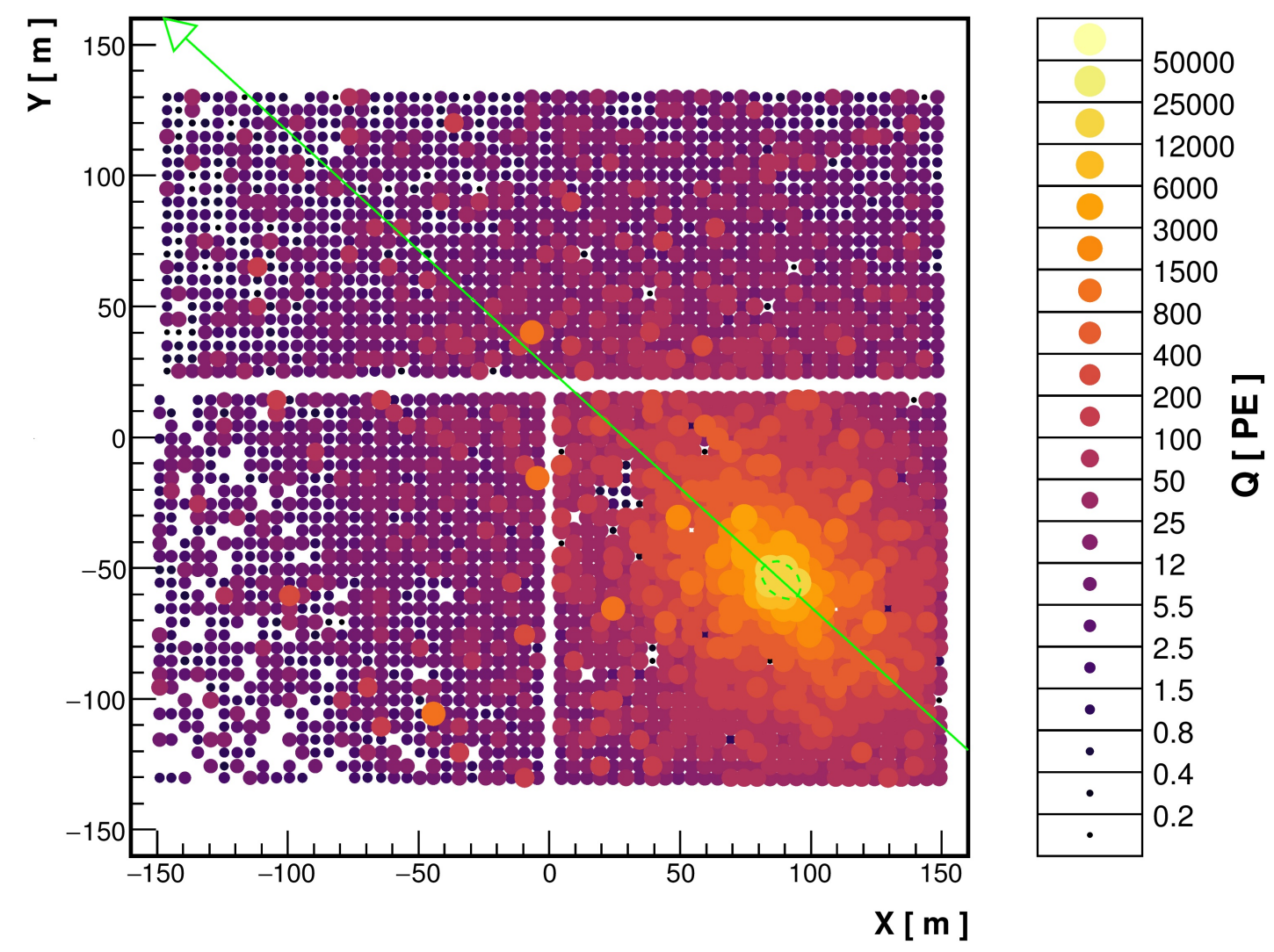

Figure 1: A high energy event detected by WCDA full array.

The one square kilometer array(KM2A) consists of 5216 electromagnetic particle detectors (EDs) and 1188 muon detectors (MDs). This array is mainly designed for $\gamma$ rays and CRs above tens of $\mathrm{TeV}$. The EDs are used to reconstruct the main parameters of air showers, such as the arrival direction and energy, as well as the core position and electromagnetic particle number in the shower, etc. The MDs are used to discriminate electromagnetic showers from hadronic showers. The ED is a scintillation detector covered by a 5-mm-thick lead plate to absorb low-energy charged particles and to convert high energy $\gamma$ rays into electron-positron pairs. The MD is a water Cherenkov detector, a tank of $36 \mathrm{~m}^{2}$ with pure water filled in. Each MD is covered by overburden soil of $2.5 \mathrm{~m}$ thick, which absorbs most of the secondary electron/positrons and $\gamma$ rays in showers. More details about the detectors are presented in [3]. The full-KM2A array was completely deployed and started operation since July 2021. A typical event recorded by KM2A is shown in Fig.2.

WFCTA has 18 Cherenkov telescopes. Each Cherenkov telescope consists of an array 

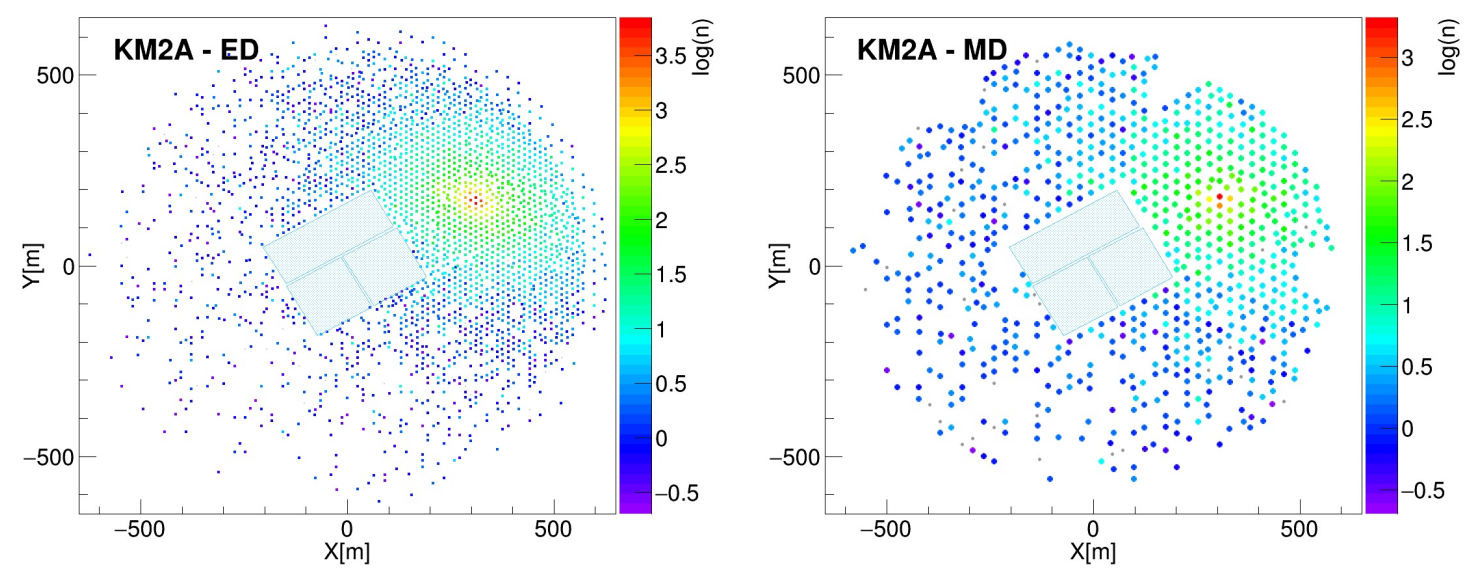

Figure 2: A typical event detected by full KM2A, these two maps show the triggered EDs and MDs at the same time.

of $32 \times 32 \mathrm{SiPMs}$ and $\mathrm{a} \sim 5 \mathrm{~m}^{2}$ spherical aluminized mirror. It has a field of view (FOV) of $16^{\circ} \times 16^{\circ}$ with a pixel size of approximately $0.5^{\circ} \times 0.5^{\circ}$. In the first observation stage, the main axis of six telescopes are arranged to point at the zenith angle of $30^{\circ}$, and cover $22^{\circ}$ to $38^{\circ}$ in zenith angle and $0^{\circ}$ to $156^{\circ}$ in azimuth angle. WFCTA, WCDA- 1 and muon detectors in KM2A are combined together as a calorimeter-like complex detector to measure air shower energy and composition. The spectra of proton and light component around the knee will be measured using the data taken in the first observation stage. More han 750 hours of shower data are collected and under analysis. In the second observation stage, the main axis of eighteen telescopes are arranged to point at the zenith angle of $45^{\circ}$, and cover $37^{\circ}$ to $53^{\circ}$ in zenith and $0^{\circ}$ to $360^{\circ}$ in azimuth. WFCTA and KM2A are combined to measure the energy spectra of iron and heavy component around the iron knee. The first six telescopes are located at the southwest corner of WCDA-1, and has started operation since October 2019. Now, the second stage of operation has started since July 2021. A typical event recorded by WFCTA is shown in Fig.3.
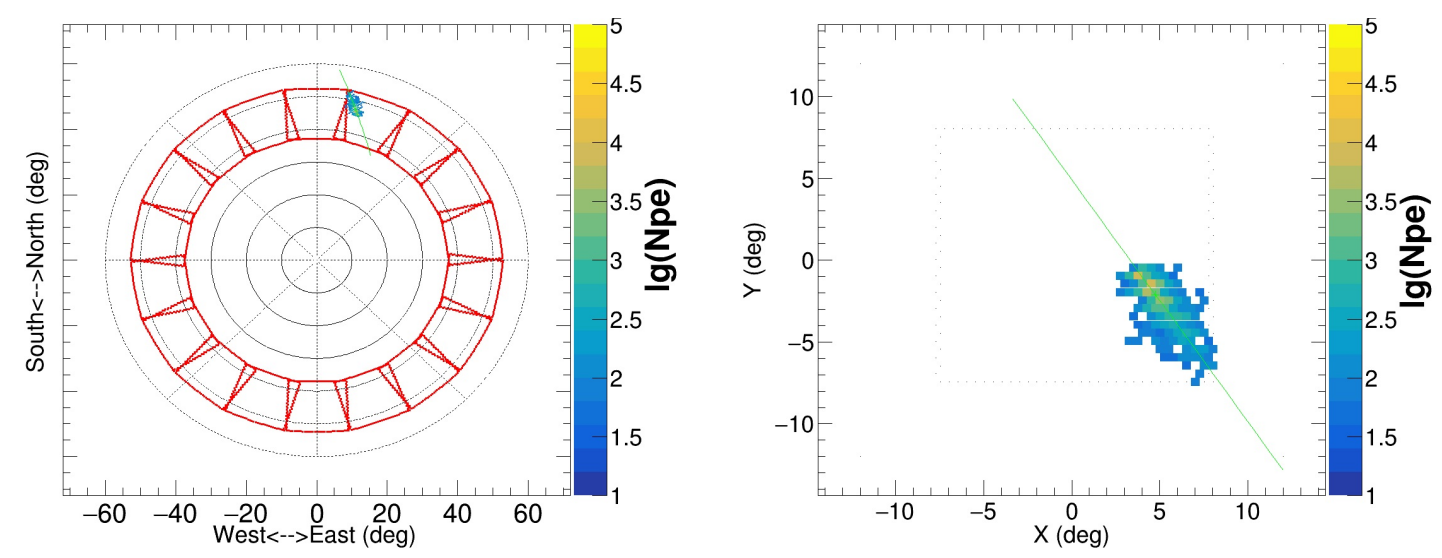

Figure 3: A typical event from WFCTA full array. 


\section{Gamma-ray Astronomy Science}

\subsection{Crab Nebular observation}

The Crab Nebula is a bright source with steady $\gamma$-ray emission powered by the Crab Pulsar's rotational energy, and well known as a standard candle for $\gamma$-ray astronomy. LHAASO has detected $\gamma$ rays from the Crab Nebula with high significance by both KM2A and WCDA [4] [5], remarkably, LHAASO has also achieved the Crab energy spectrum over three energy decades from $0.5 \mathrm{TeV}$ to $1.1 \mathrm{PeV}$ [6].

The Crab detection is important to validate the performance of KM2A and WCDA, specifically to study the detector response to $\gamma$ rays. KM2A is mainly designed to observe a large fraction of the northern sky to hunt for $\gamma$-ray sources at energies above $10 \mathrm{TeV}$. Detailed studies of the performance of first operation stage with half-KM2A have been carried out by Monte Carlo simulations. At $100 \mathrm{TeV}$ with zenith angle less than $35^{\circ}$, the angular and shower core resolution are about $0.26^{\circ}$ and $3 m$, respectively. The particle density with distance $<50 \mathrm{~m}$ to the shower core (denoted as $\rho_{50}$ ), obtained by fitting the lateral distribution with Nishimura-Kamata-Greisen (NKG) function, is used to estimate the $\gamma$-ray energy. For energy above $100 \mathrm{TeV}$, the energy resolution is better than $20 \%$ refering to the Gaussian-like energy resolution function.

KM2A has an excellent $\gamma$-ray/background (mainly CRs) discrimination power by using the underground muon detectors (MDs). Counting number of measured muons in a shower is crucial to discriminate the muon-poor $\gamma$-ray induced shower from muon-rich CR-induced shower. Therefor, the ratio between measured muons $\left(N_{\mu}\right)$ by MDs and electrons $\left(N_{e}\right)$ by EDs is used to discriminate primary $\gamma$-rays from cosmic nuclei. Fig. 4 shows the detection rate of the CR-induced showers within a cone of $1^{\circ}$ centered at the Crab. The muon-cut filter requires that the number of $N_{\mu}$ detected by MDs in the shower must be less than $1 / 230$ of $N_{e}$ detected by EDs. The cut reduces the CR background by factors of 1000 and 500,000 at $50 \mathrm{TeV}$ and $1 \mathrm{PeV}$, respectively. And the right panel of Fig. 4 shows a total significance of Crab $(>25 \mathrm{TeV})$ with half-KM2A data collected from 11 Sep 2019 to 7 Jul 2020. The pointing accuracy of half-KM2A is thus obtained by fitting Crab position which is $<0.1^{\circ}$.

WCDA is designed to perform deep surveys for very-high-energy $\gamma$-ray sources. The first Water Cherenkov detector WCDA-1 has been operating since April 2019. A performance of WCDA-1 has been studied by observing the Crab Nebula as the standard candle. The arrival direction and shower core position of primary $\gamma$-ray events are reconstructed and verified by using the simulated events. The shower core location has a resolution of better than $3 \mathrm{~m}$ for energetic events of $10 \mathrm{TeV}$ and above. The angular resolution for the $\gamma$-ray arrival direction is better than $0.4^{\circ}$ above $3 \mathrm{TeV}$ according to the well-measured detector orientation and timing calibration for each detector unit. For energy estimation, the number of triggered 8-inch PMT units $N_{\text {hit }}$ is selected as a shower energy estimator. A Monte Carlo simulation of the shower development is carried out to establish the energy response function. The energy resolution is defined as a symmetric Gaussian function, and the energy resolution is found to be $33 \%$ for $\gamma$ rays above $6 \mathrm{TeV}$. 

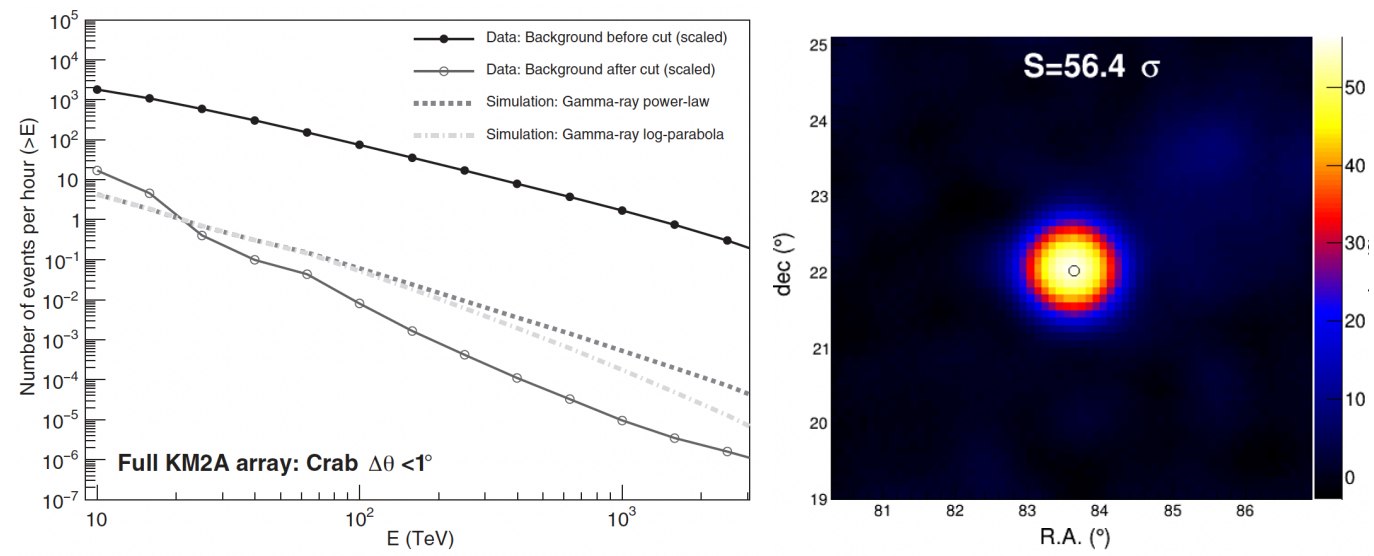

Figure 4: Left panel: the rates of detection of $\gamma$ rays from the Crab and the CR background events above the shower energy $\mathrm{E}$ by KM2A in a cone of $1^{\circ}$ centered at the Crab direction [6]. Right panel: the significance map of Crab Nebular observed by half-KM2A.
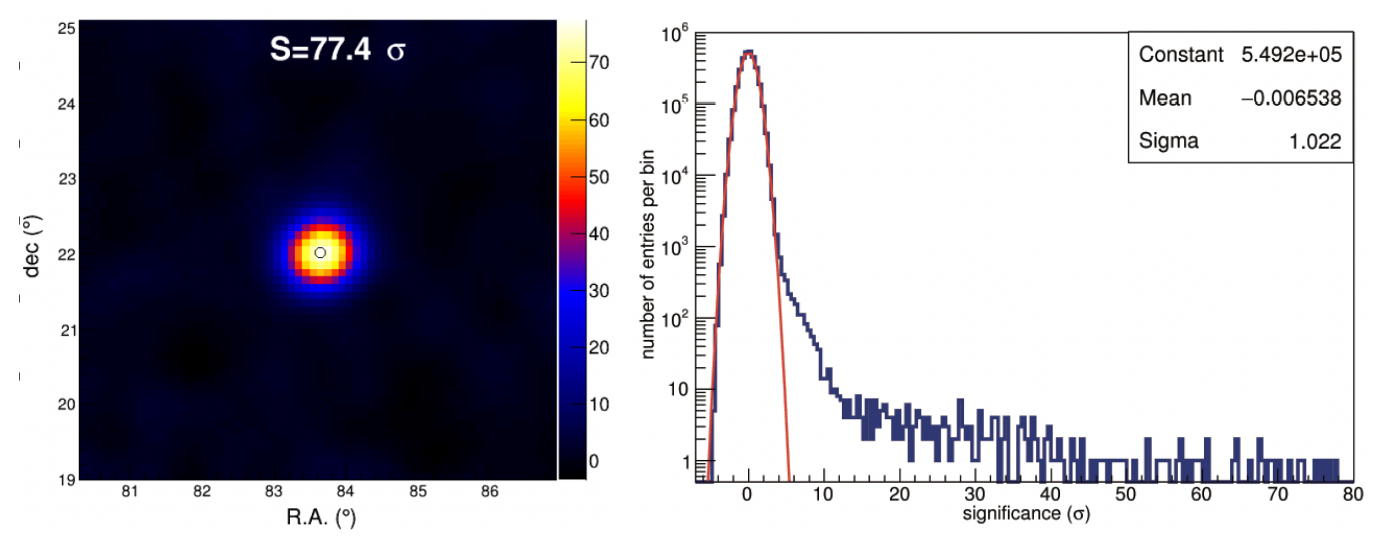

Figure 5: Left panel: Two-dimensional significance map around the position of the Crab Nebular; Right panel: the distribution of the significance among $3600 \times 900$ bins [4] using WCDA- 1 data up to 11 March 2020.

To maximize the sensitivity to $\gamma$-ray showers, the cosmic-ray background suppression is performed by characterizing the features of showers induced by different primary particles, so a parameter named compactness is employed in WCDA-1. Consequently, the cosmicray background rejection rate is $97.7 \%$ around $1 \mathrm{TeV}$ and $99.8 \%$ around $6 \mathrm{TeV}$ with an approximate photon acceptance of $50 \%$ after applying the compactness-based cut to separate gamma-induced showers from CR-induced showers. Crab Nebular observation with WCDA-1 is carried out with all events collected in the period from 19 Apr 2019 to 11 Mar 2020. The significance map around Crab Nebula and distribution of the events as a function of the significance are shown in Fig. 5. A clear Crab image with a significance of $77.4 \sigma$ has been achieved. The pointing accuracy of WCDA-1 as a gamma ray telescope is better than $0.1^{\circ}$ above $1 \mathrm{TeV}$.

The spectral energy distribution(SED) of Crab Nebula in the range from $500 \mathrm{GeV}$ to 1.1 $\mathrm{PeV}$ is determined by combining WCDA and KM2A data, shown in Fig. 6. This spectrum 


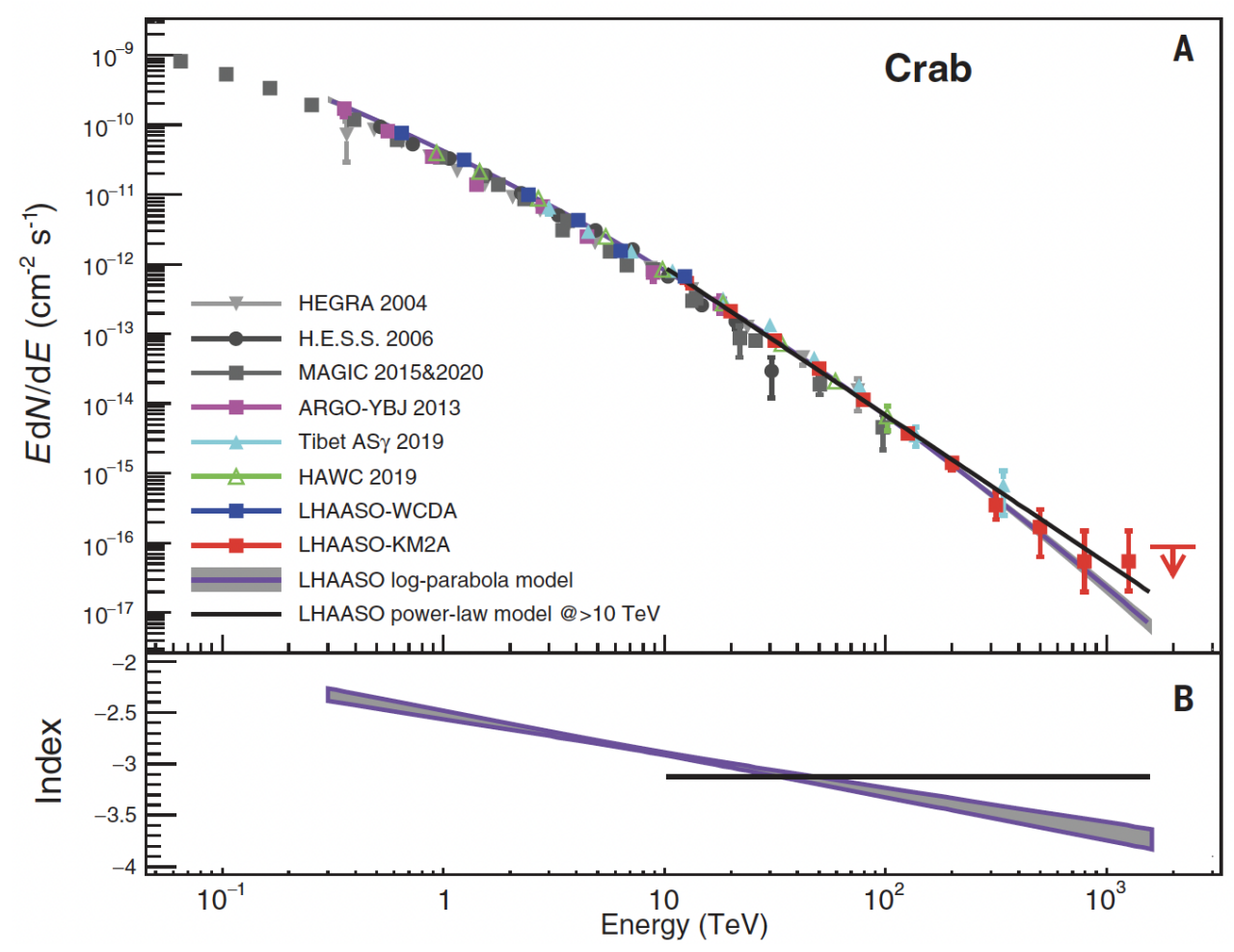

Figure 6: $\gamma$-ray flux of Crab Nebular measured by LHAASO and spectral fitting.

shows a gradual steepening over three energy decades and agreeing with other experiments below $100 \mathrm{TeV}$. The consistent between WCDA and KM2A around the overlapping energies is also approved as shown in the spectrum. LHAASO is thus recognized to be a unique experiment to get an accurate ultra-high-energy(UHE) SED of Crab. The measured 1.1 $\mathrm{PeV}$ photon provides direct evidence for the acceleration of $2.3 \mathrm{PeV}$ electron in the source. According to this measurement result, the acceleration efficiency of electron can at least reach to $15 \%$ of the upper limit according to the classical electrodynamics and ideal magnetohydrodynamics theory, thus surpassing the acceleration efficiency of supernova shock by a factor of 1,000. This poses challenges to the standard paradigm of electron acceleration in high-energy astrophysics, we reported this result in Ref. [6].

\subsection{UHE Sky Survey}

With less than one year operation of partly completed KM2A, there are many hot spots as clusters of $\gamma$ rays in specific directions of the sky [7]. By using a point-like template for the Crab Nebula and LHAASO J2108 +5157 and $0.3^{\circ}$ extension templates for other bright sources, we detected more than 530 photons in 12 multiple UHE $\gamma$-ray sources at energies $\geq 100 \mathrm{TeV}$ with statistical significance $\geq 7 \sigma$, and they are all located in Galactic plane, shown in Fig. 7. The acceleration of protons to $\mathrm{PeV}$ energies requires extreme physical conditions, and these 12 UHE sources detected by LHAASO leave an open window to the origin of extreme accelerators. 

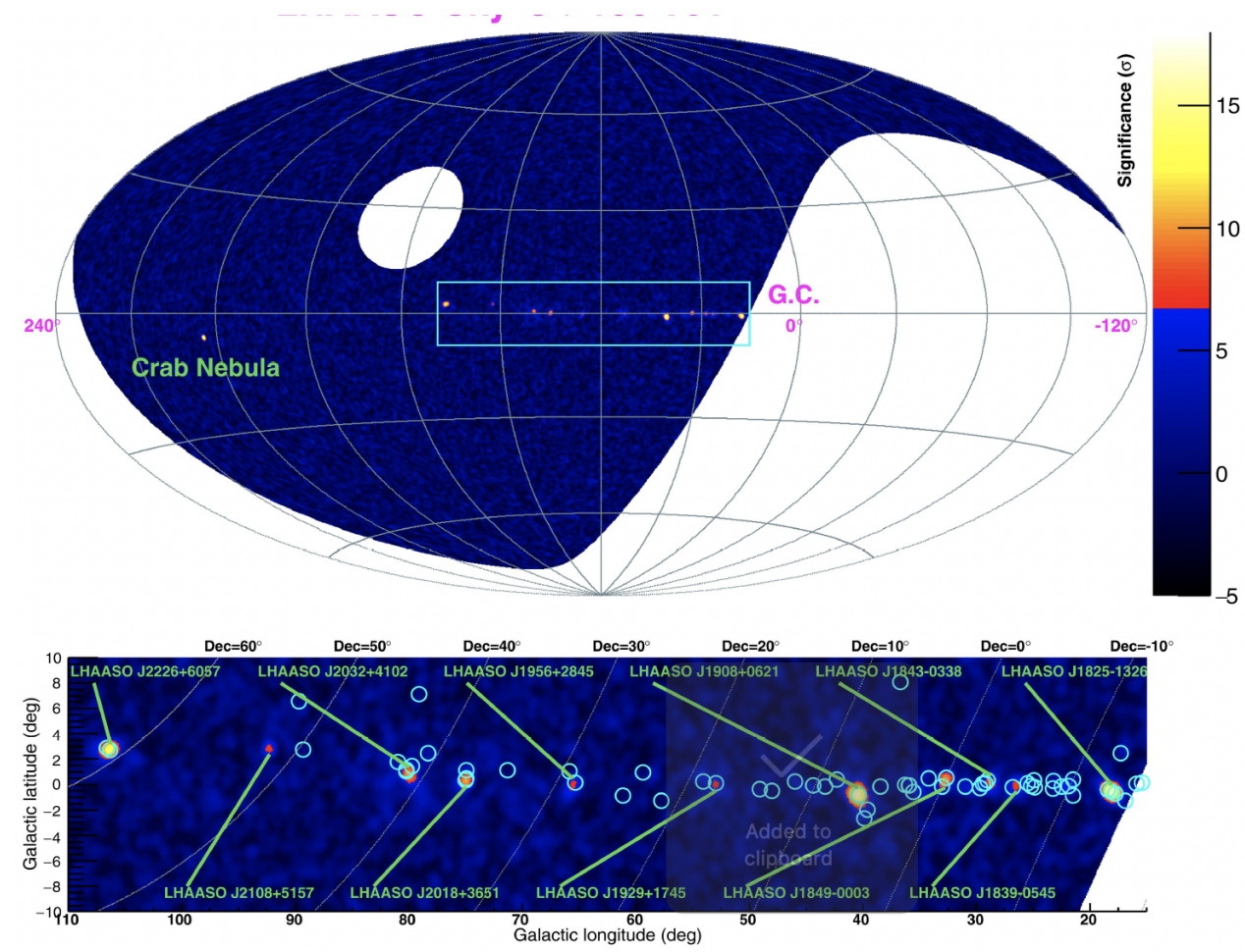

Figure 7: LHAASO sky map at energies above $100 \mathrm{TeV}$. The circles indicate the positions of known very-high-energy $\gamma$-ray sources.

Above $400 \mathrm{TeV}$, KM2A measures $\gamma$ rays essentially background-free, and every single photon has its own energy measured independently. A photon with reconstructed energy higher than $1.4 \mathrm{PeV}$ in the $1^{\circ}$ cone centred at the source location is detected from the Cygnus region, the triggered map of this event is shown in Fig. 8. For this highest-energy photon from the Cygnus region, the measured ratio $N_{\mu} / N_{e}=1 / 941$ rejects almost all CRs, thus estimates the chance probability of $0.03 \%$ to mis-identified as a gamma ray event. This event might be an evidence of the operation of a hadronic PeVatrons in the massive star cluster. Adequate photon statistics provided by LHAASO for spectrometric and morphological studies of this object, which is located in a rather complex region crowded with several competing sources, is foreseen as crucial evidence in upcoming 1 or 2 years.

\subsection{Galactic Sources}

\subsubsection{LHAASO J2032 +4102}

The UHE source LHAASO J2032+4102 is positionally overlapped with Cygnus Cocoon. The significance map around Cygnus cocoon region above $25 \mathrm{TeV}$ is shown in Fig.9, and a two-dimension Gaussian model with sigma fixed at $0.3^{\circ}$ is used here. The maximum significance is more than $20 \sigma$. The center of the emission is close to both the PWN and OB2 cluster. Due to the complex composition of this region, a multi-source fitting procedure was adopted to fit the emission in this region simultaneously. The emission is well described by three sources:A slightly extended source with Gaussian width of $0.24^{\circ} \pm 0.03^{\circ}$ at the center 


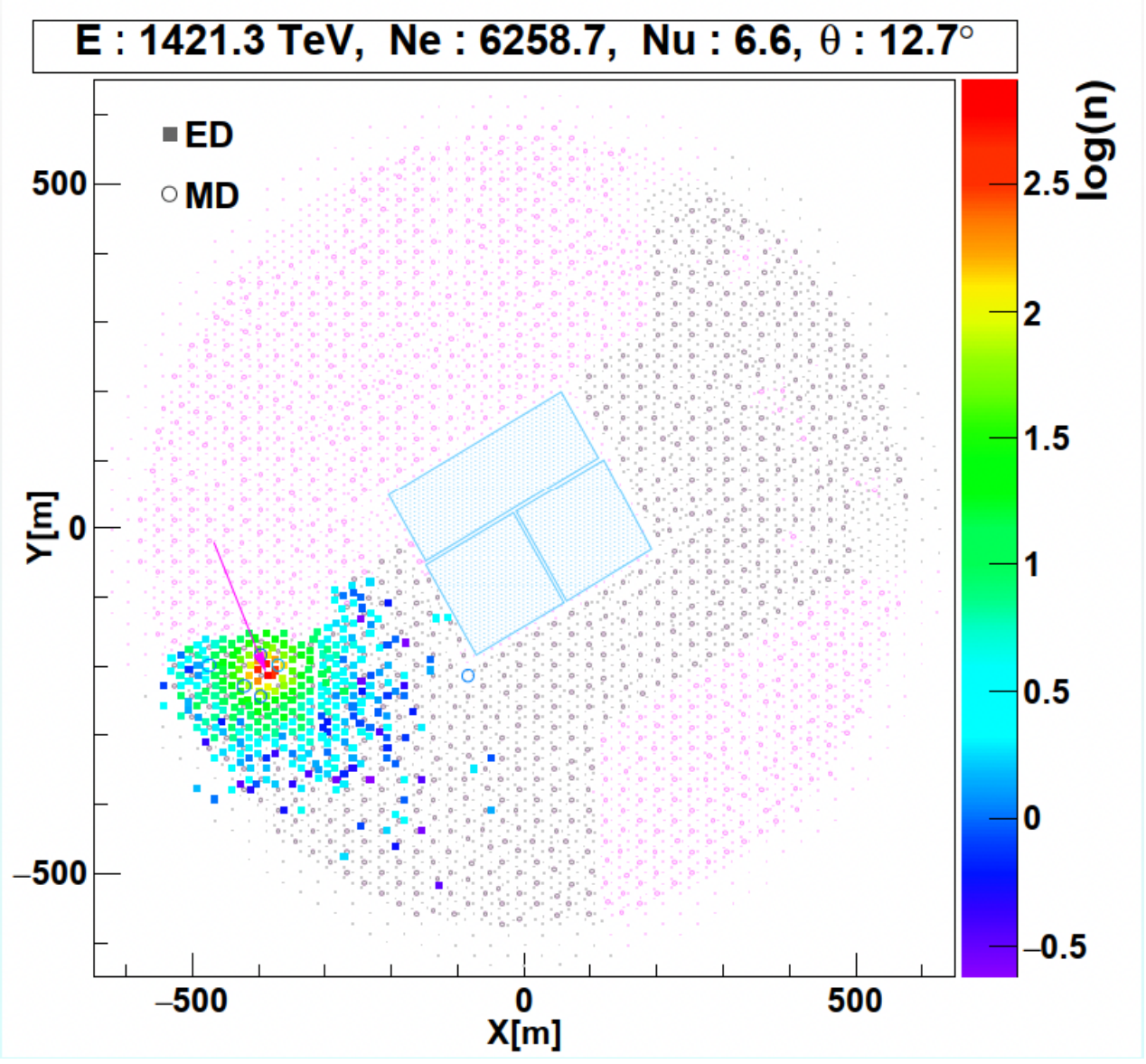

Figure 8: The $\gamma$-ray event with $1.4 \mathrm{PeV}$ from Cygnus region.

of the region $\left(\mathrm{RA}=307.98^{\circ} \pm 0.03^{\circ}\right.$, Dec $\left.=41.42^{\circ} \pm 0.03^{\circ}\right)$, which maybe associated with the PWN TeV J2032+4130. A very extended source with Gaussian width of $2.8^{\circ} \pm 0.3^{\circ}$ and best fitted position at $\mathrm{RA}=308.2^{\circ} \pm 0.4^{\circ}$ and $\mathrm{DEC}=41.3^{\circ} \pm 0.3^{\circ}$, which is the counterpart of $\mathrm{GeV}$ Cygnus Cocoon(shown in Fig.9). The third source is at $\mathrm{RA}=305.5^{\circ} \pm 0.2^{\circ}$ and $\mathrm{DEC}=40.4^{\circ} \pm 0.1^{\circ}$, which maybe associated with Gamma Cygni.

\subsubsection{LHAASO J1825-1326}

LHAASO J1825-1326 (Figure. 10) is detected at an 18.0 $\sigma$ level above $25 \mathrm{TeV}$ with an extension of $\sigma=0.30^{\circ} \pm 0.06^{\circ}$. The source has a spectrum fitted by a log-parabola with $a=0.92, b=1.19\left(A I C_{L O G}=11.6\right)$ or a power-law with $\Gamma=3.36\left(A I C_{P L}=14.8\right)$. There are two energetic pulsars, PSR J1826-1334 and PSR J1826-1256, located in the source region, which may be the energy sources of the UHE emission. In the VHE $\gamma$-ray regime, HESS detected an energy dependent morphology which is consistent with the leptonic origin of the $\gamma$-ray emission [7]. 


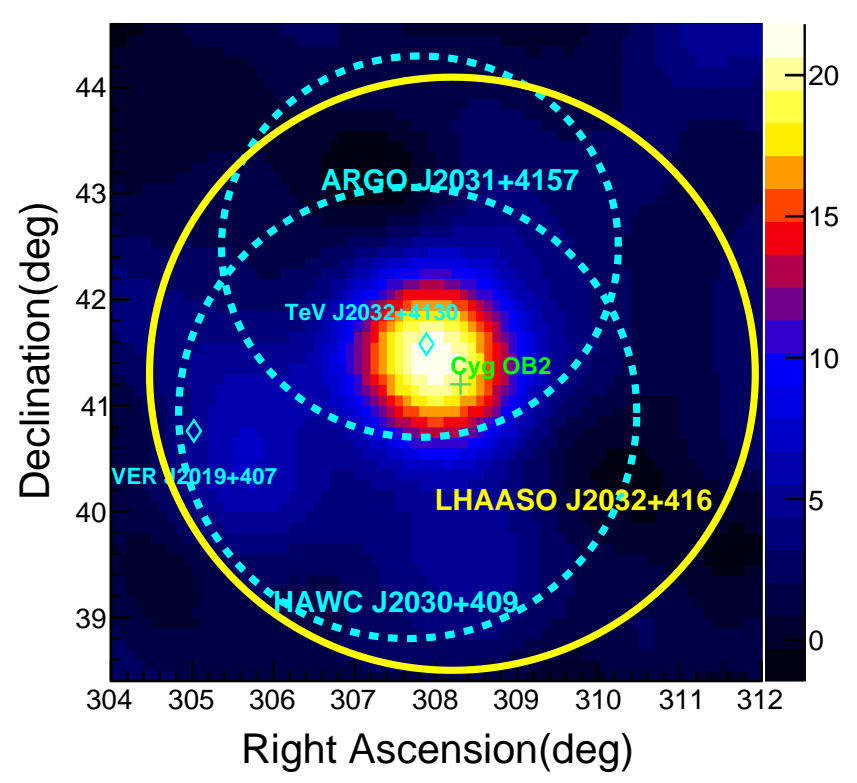

Figure 9: The significance map in Cygnus Cocoon region above $25 \mathrm{TeV}$. The blue diamonds marks $\mathrm{TeV}$ sources $\mathrm{TeV} \mathrm{J} 2032+4130$ and VER J2019+407. The two blue dashed circle marks two very extended sources ARGO J2031+4157 and HAWC J2030+409. The yellow circle marks the source LHAASO J3032+416.

\subsubsection{LHAASO J1908+0621}

LHAASO J1908+0621 is consistent with the MGRO J1908+06 which was discovered by the MILAGRO collaboration [8] and later confirmed by the HESS atmospheric Cherenkov telescope [9]. It spatially associated with a middle-aged supernova remnant (SNR) G40.50.5 and an energetic $\gamma$-ray pulsar PSR J1907+0602 2021nature. The age of G40.05-0.5 is estimated between 20 and $40 \mathrm{kyr}$ and the distance is uncertain. The PSR J1907+0602 has a characteristic age of $19.5 \mathrm{kyr}$ and a spin-down luminosity of $\sim 3 \times 10^{36} \mathrm{erg} \mathrm{s}^{-1}$. The distance of PSR J1907+0602 was estimated to be $3.2 \mathrm{kpc}$. The nature of MGRO J1908+06 remains unrevealed and a single accelerator cannot explain the whole set of multi-wavelength data.

The significance map around LHAASO J1908+0621 with $E \geq 25 \mathrm{TeV}$ are shown in Figure 11. We use the two-dimensional Gaussian model template to study the morphology of the source. The centroid of $\gamma$-ray emissions with energies above $25 \mathrm{TeV}$ is R.A. $=287.04^{\circ} \pm$ $0.03^{\circ}$ (stat), Dec. $=6.24^{\circ} \pm 0.04^{\circ}$ (stat) $(\mathrm{J} 2000)$ and the extension is $\sigma=0.57^{\circ} \pm 0.03^{\circ}$. Taking into account the Gaussian extension of $0.57^{\circ}$, the resulting differential flux can be fitted by a log-parabola for $a=2.27, b=0.46$ and a power-law for $\Gamma=2.89$. The Akaike Information Criterion (AIC) of log-parabola and power-law are 15.1 and 30.1.

The $\gamma$-ray spectral points with energies from $\mathrm{GeV}$ to several hundred $\mathrm{TeV}$ could be explained by accelerated electrons following a spectrum of $N(E) \propto N_{e}^{-1.75} \exp \left\{-E_{e} /(800 \mathrm{TeV})\right\}$. Alternatively, the $\gamma$-ray emission could be produced by protons accelerated up to $\mathrm{PeV}$ colliding with the ambient dense gas. The spectrum of accelerated protons has a complex form which is a broken power law with an exponential cutoff, with indices 1.2 and 2.7 below and 

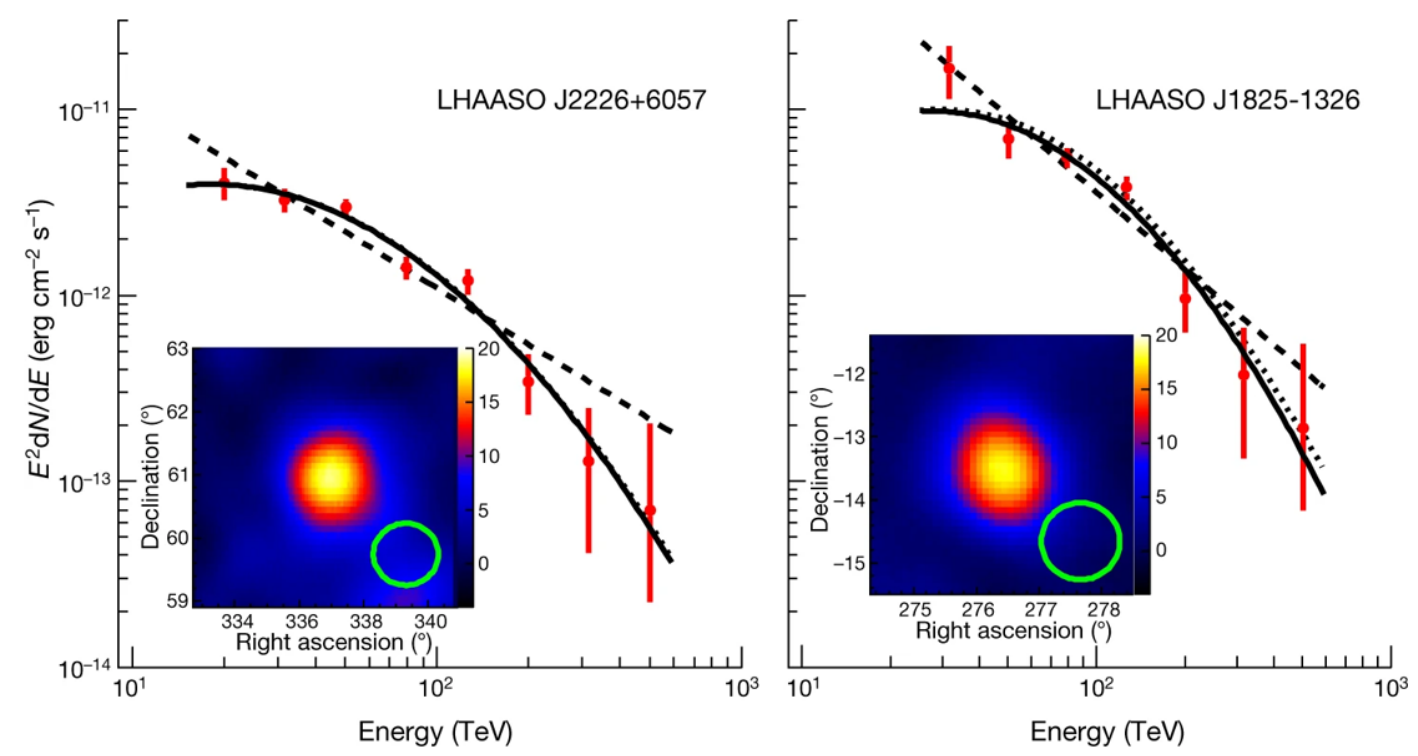

Figure 10: Spectral energy distributions and significance maps of LHAASO J2226+6057 and LHAASO J1825-1326 [7]

above $25 \mathrm{TeV}$ and a cutoff energy of $1.3 \mathrm{PeV}$.

\subsubsection{LHAASO J2108+5157}

LHAASO J2108 +5157 is newly discovered by LHAASO at approximately $9.5 \sigma$ and $8.5 \sigma$ significance level in both energy bands of $25-100 \mathrm{TeV}$ and $>100 \mathrm{TeV}$, respectively [7]. This source is not significantly favored as an extensive source with the angular extension smaller than the point-spread function of KM2A. An upper limit on the extension of the source is calculated to be $0.26^{\circ}$ at $95 \%$ confidence level (CL). Its spectrum from $20 \mathrm{TeV}$ to $500 \mathrm{TeV}$ is characterized by a power-law with index $-2.83 \pm 0.18$ (stat). No obvious counterparts were found in the region of LHAASO J2108+5157 at other wavelengths. It is correlated with the molecular cloud [MML2017]4607. The UHE $\gamma$ rays could be explained by interactions of protons with the ambient gas through the production and decay of $\pi^{0}$ mesons (As shown in Figure 12). The energy spectrum of protons follows a power-law with an exponential cutoff: $N(E) \propto N_{p}^{-2} \exp \left\{-E_{p} /(600 \mathrm{TeV})\right\}$. Other possible scenarios, such as a PWN, can also be invoked to explain the KM2A observed $\gamma$ rays. The primary electron spectrum follows a power-law with an exponential cutoff: $N(E) \propto N_{e}^{-2.2} \exp \left\{-E_{e} /(200 \mathrm{TeV})\right\}$. Because of the absence of pulsar counterpart, the PWN scenario remains uncertain. So far, no conclusion about the origin of its UHE emission can be achieved.

\subsubsection{LHAASO J2226 +6057}

LHAASO J2226 +6057 (Figure. 10) is detected at a significance of $18.0 \sigma$ above $25 \mathrm{TeV}$, exhibiting a log-parabola spectral for $a=1.56, b=0.88\left(A I C_{L O G}=12.3\right)$ and a power-law for $\Gamma=3.01\left(A I C_{P L}=24.4\right)$. The detected highest photon energies is $0.57 \pm 0.19 \mathrm{PeV}$. The Source is spatially associated with SNR G106.3+2.7 which is a comet-shaped radio source, 


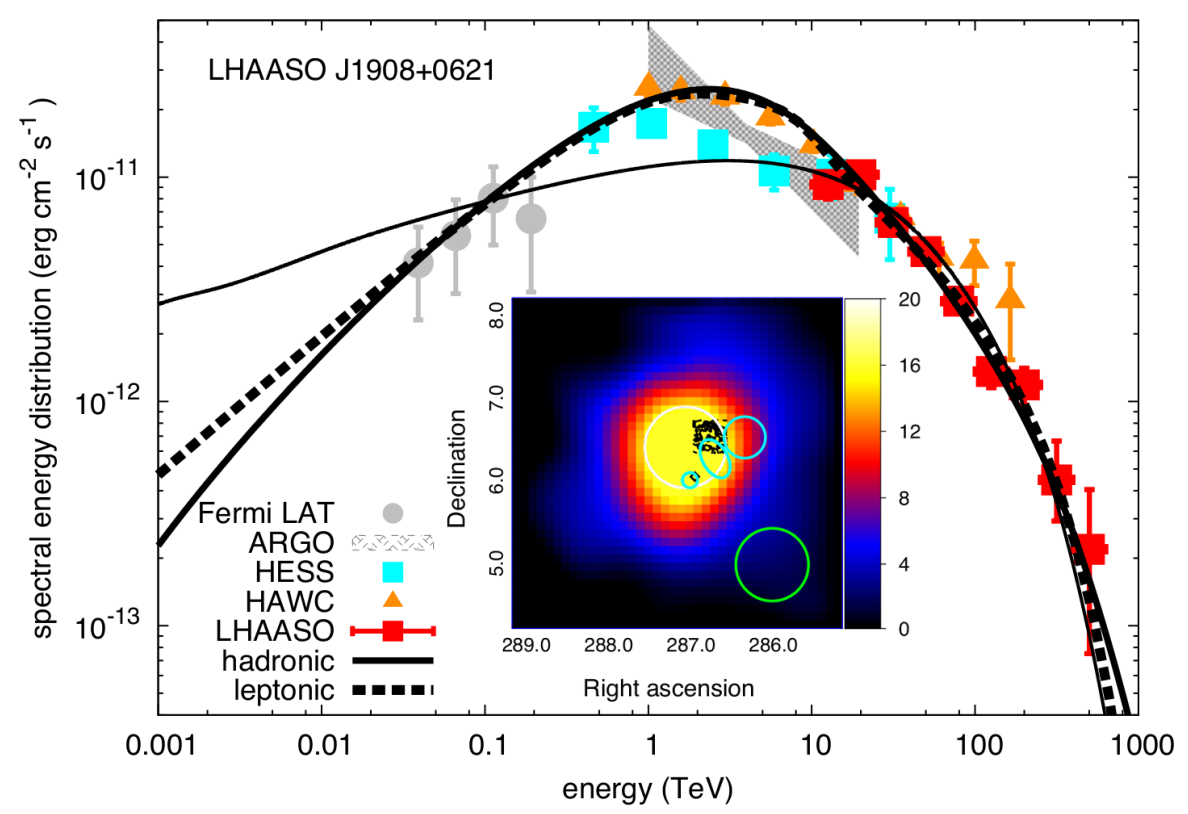

Figure 11: Phenomenological fits to the $\gamma$-ray observations of LHAASO J1908 +0621 , and previous observations of potential counterparts [7].

with a brighter 'head' and an extended 'tail' region. The 'head' region also contains an offcenter PWN in north named 'Boomerang' powered by the pulsar PSR J2229+6114, whose characteristic age and luminosity are $10 \mathrm{kyr}$ and $2.2 \times 10^{37} \mathrm{erg} \mathrm{s}^{-1}$. The very-high-energy $\gamma$-ray emission above $10 \mathrm{TeV}$ observed by $\mathrm{AS} \gamma$ is associated with a molecular cloud rather than with the pulsar PSR J2229+6114 [7]. A dedicated analysis of LHAASO J2226+6057 from $500 \mathrm{GeV}$ to $1 \mathrm{PeV}$ is undergoing. This will provide crucial information to disentangle the origin of the gamma ray emission observed.

\section{Cosmic-ray Physics Science}

LHAASO is a hybrid detector array for the purpose of precisely measure charged CR induced air showers. WCDA or ED array can measure the shower arrival direction with a resolution of $0.2^{\circ}$ and shower location with a resolution of $2 \mathrm{~m}$. WCDA can measure the energy flux in a range of $5 \mathrm{~m} \times 5 \mathrm{~m}$ around shower cores. Muon detector array can measure $\mu$ content with a dynamic range of $1-10^{4}$ muons for each MD. WFCTA can measure the atmospheric depth of the shower maximum, $X_{\max }$ with a resolution of $40 \mathrm{~g} / \mathrm{cm}^{2}$. WFCTA can also measure the shower energy with a resolution of 15\%. Multi-parameter observation of air showers allows us to measure the CR spectrum, elemental composition and anisotropy with high resolution, which would be useful in searching for the origin of ultra-high energy CRs, and improving the study of their acceleration and propagation in the Milky Way. 


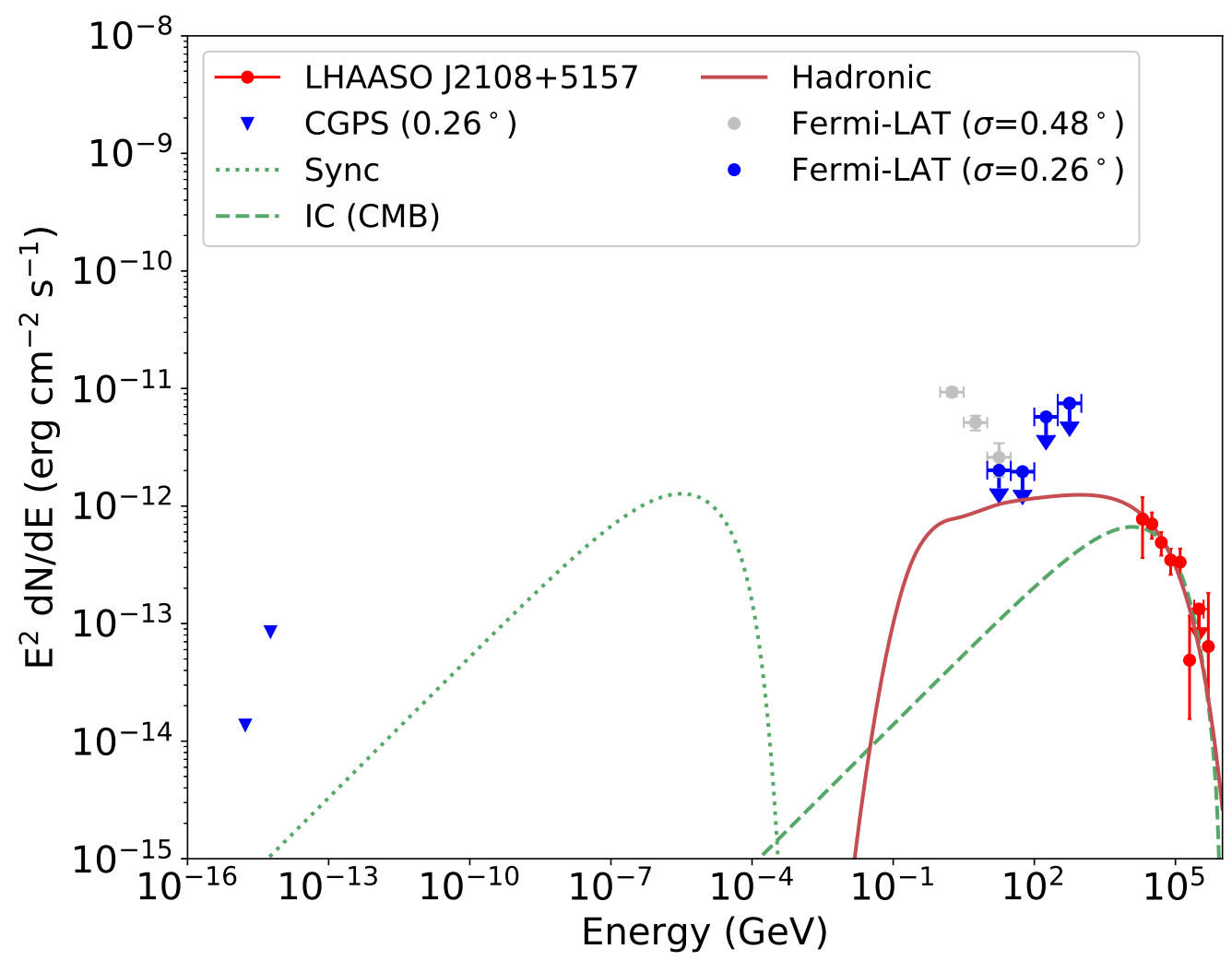

Figure 12: The multiwavelength SEDs of LHAASO J2108+5157 with hadronic and leptonic modeling [7]. The red points and arrows are the LHAASO-KM2A observations. The blue triangles are the radio fluxes. The grey points and blue arrows are the Fermi-LAT spectral points and upper limits.

The combination of WFCTA and WCDA is good for measuring the energy spectra of the $\mathrm{CR}$ species over a very wide energy range from $1 \mathrm{TeV}$ to $10 \mathrm{PeV}$, in which the knees of spectra of protons and Helium nuclei are expected. The precise measurement of the knee structures is important and requires the separation of proton showers from all other CR showers, and $\mathrm{H}+\mathrm{He}$ mixed samples from all other heavier nuclei induced showers, respectively. This is under investigation still. The combination can provide an absolute energy scale calibration with a proven technique of measuring the westward shift of the Moon shadow in the galactic CRs due to the geomagnetic field (GMF) [10]. The displacement of the center of the shadow is proved to be inversely proportional to the cosmic-ray energy[11].

In LHAASO experiment, the precise measurement of Moon shadow shift by WCDA allows us to calibrate its energy scale up to $35 \mathrm{TeV}$. The scale can be propagated to WFCTA by using the commonly triggered CR events by the two detector arrays. In current operation phase, WCDA-1 data from May 1st, 2019 to Jan. 31th, 2020 has been collected, and analyzed for measuring the cosmic-ray energy scale. The total effective observation time is $\simeq 731.2$ hours with zenith angles $\leq 45^{\circ}$ for the Moon observation, and the total number of events is about $\simeq 4.17$ million with arrival direction $<5^{\circ}$ to the Moon position. To achieve the energy calibration with WCDA-1, we need to (1) measure the Moon shadow shift as a 
function of the total number of photo-electron $N_{p e}$ as a shower energy estimator, i.e., the displacement $\triangle$ vs. $N_{p e} ;(2)$ set up the function between the displacement and primary CR energy by tracing CRs with certain composition through GMF, $\Delta=1.59^{\circ} /(E / Z)$, where $E$ is the particle energy in $\mathrm{TeV}$ and $Z$ is the charge of nucleus in electron charge $e$; (3) solve the average rigidity of $\mathrm{CR}$ events that trigger WCDA-1 in the relevant ranges of $N_{p e}$ by using the air shower and detector response simulation, as well as the flux ratio between $\mathrm{H}$ and He, the function of displacement $\triangle=2.1 / E_{W C D A}$ is obtained, where $E_{W C D A}$ is the median energy in $\mathrm{TeV}$ of the $\mathrm{CR}$ showers that have $N_{p e}$ in the corresponding intervals. So, in the energy range from $6.6 \mathrm{TeV}$ to $35.0 \mathrm{TeV}, N_{p e}$ can be used as an energy proxy according to the established relation $E_{W C D A}=b\left(N_{p e}\right)^{\beta}$, where $b=1.33_{-1.08}^{+5.29}$, and $\beta=0.95_{-0.17}^{+0.18}$, as shown in Fig. 13, with the deflecting angel $\Delta$ as the bridge.

A set of commonly triggered events is used to propagate the absolute energy scale of WCDA-1 to WFCTA, specifically by collecting the data observed at 10 nights in Jan 2020 with moonless and very excellent weather conditions. In order to achieve a more precise energy reconstruction, the shower cores are required to locate inside the WCDA-1, as well as the full Cherekov image should be measured by applying a telescope FOV cut. The median energy reconstructed by WFCTA is found to be $21.9 \mathrm{TeV}$ as shown in the left panel in Fig. 13 in red and the median energy determined by WCDA-1 is 23.4 for the same bulk of events as in black in the left panel in Fig. 13. This indicates that the energy scale of $21 \pm 6$, measured by WCDA-1, can be propagated to WFCTA with a uncertainty of $7 \%$. However, the uncertainty of the measured energy scale of $30 \%$ is largely dominated by the low statistics of events using in the Moon shadow position measurement, which would be improved by accumulating longer observation time in future. For showers with greater energy, the energy reconstruction totally relies on the simulation of air showers and detector response of WFCTA. More details about the energy scale calibration can be reached in [12].

\section{Acknowledgement}

This work was supported in China by the National Key R\&D program of China under grants 2018YFA0404201, 2018YFA0404202, 2018YFA0404203, and 2018YFA0404204; by NSFC under grant nos. 12022502, 11905227, U1831208, U1931112, 11635011, 11761141001, 11905240, 11675204, 11475190, U2031105, and U1831129; and in Thailand by grant RTA6280002 from Thailand Science Research and Innovation. Chengdu Management Committee of Tianfu New Area provided financial support for research with LHAASO data.

\section{References}

[1] E. G. Berezhko, S. P. Knurenko, and L. T. Ksenofontov. Chin. Phys. C, 36.1 (2012),31-6

[2] Veniamin Berezinsky, Askhat Gazizov, and Svetlana Grigorieva. Phys. Rev. D 74 (2006), 043005

[3] Huihai He. Radiation Detection Technology and Methods 2 (2018)

[4] F. Aharonian et al. Chin. Phys. C 45.8 (2021), 085002. 

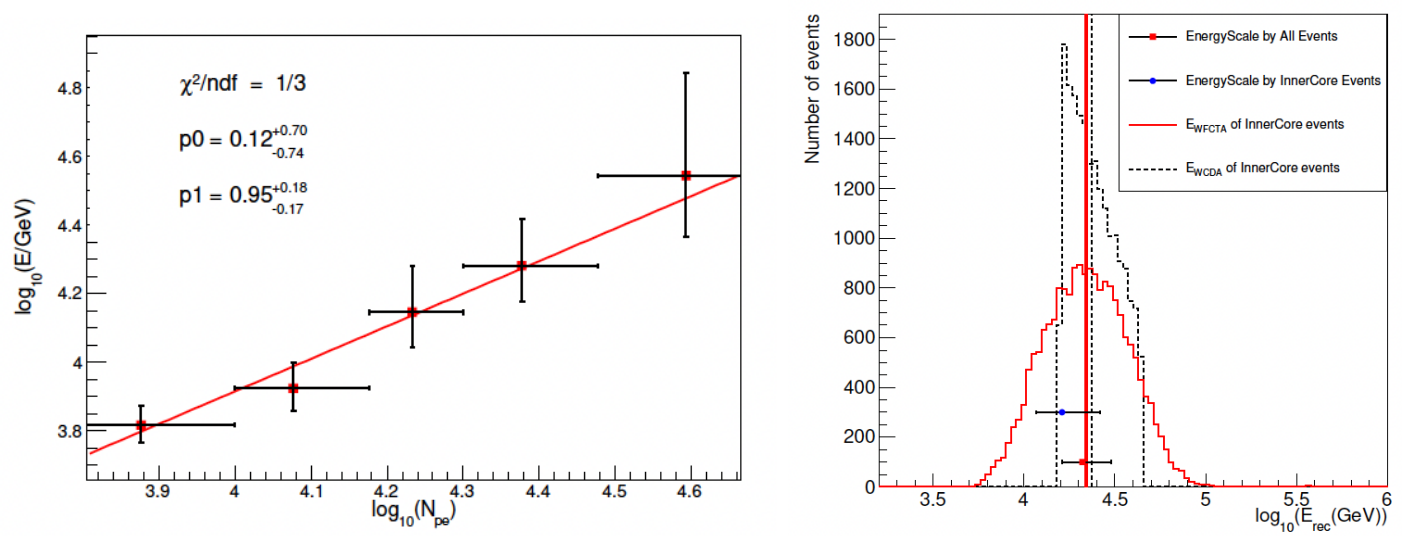

Figure 13: Left pad: the average shower energy measured using the Moon shadow shift versus $N_{p e}$, the total number of photo-electrons detected by WCDA-1 detector. Right pad: Distribution of reconstructed energies for the commonly triggered events, the red solid lines show the distribution of energies reconstructed by WFCTA [12] and its median energy. The dotted lines show the distribution of energies reconstructed by WCDA and its median energy. Red and blue dots show the measured energy scales by using the shifts of the Moon shadows with different cuts. The rather large uncertainties of the scales is mainly due to the statistical error of the shift measurements.

[5] F. Aharonian et al. Chin. Phys. C 45.2 (2021), 025002

[6] Zhen Cao et al. Science (2021)

[7] Zhen Cao et al. Nature 7594 (2021),33-6.

[8] A. A. Abdo et al. The Astrophysical Journal, 664.2 (July 2007), L91-94

[9] F. Aharonian et al. A\&A, 499.3 (June 2009),723-28

[10] B. Bartoli et al. Phys. Rev.D 92.9 (2015), 092005

[11] N. A. Tsyganenko. J. Geophys. Res. 100 (1995), 5599-612.

[12] F. Aharonian et al. Phys. Rev. D 104.6 (2021),062007 


\section{Full Authors List: LHAASO Collaboration}

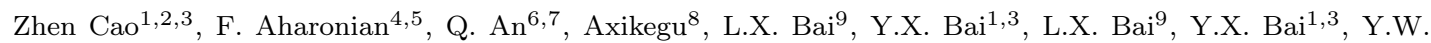
Bao $^{10}$, D. Bastieri ${ }^{11}$, X.J. Bi ${ }^{1,2,3}$, Y.J. Bi ${ }^{1,3}$, H. Cai ${ }^{12}$, J.T. Cai ${ }^{11}$, Zhe Cao ${ }^{6,7}$, J. Chang ${ }^{13}$, J.F. Chang ${ }^{1,3,6}$, B.M. Chen $^{14}$, E.S. Chen ${ }^{1,2,3}$, J. Chen ${ }^{9}$, Liang Chen ${ }^{1,2,3}$, Liang Chen ${ }^{15}$, Long Chen ${ }^{8}$, M.J. Chen ${ }^{1,3}$, M.L. Chen ${ }^{1,3,6}$, Q.H. $\mathrm{Chen}^{8}$, S.H. Chen ${ }^{1,2,3}$, S.Z. Chen ${ }^{1,3}$, T.L. Chen ${ }^{16}$, X.L. Chen ${ }^{1,2,3}$, Y. Chen ${ }^{10}$, N. Cheng ${ }^{1,3}$, Y.D. Cheng ${ }^{1,3}$, S.W. Cui $^{14}$, X.H. Cui ${ }^{17}$, Y.D. Cui ${ }^{18}$, B. D'Ettorre Piazzoli ${ }^{19}$, B.Z. Dai ${ }^{20}$, H.L. Dai ${ }^{1,3,6}$, Z.G. Dai ${ }^{7}$, Danzengluobu ${ }^{16}$, D. della Volpe ${ }^{21}$, X.J. Dong ${ }^{1,3}$, K.K. Duan ${ }^{13}$, J.H. Fan ${ }^{11}$, Y.Z. Fan ${ }^{13}$, Z.X. Fan ${ }^{1,3}$, J. Fang ${ }^{20}$, K. Fang ${ }^{1,3}$, C.F. Feng ${ }^{22}$, L. Feng ${ }^{13}$, S.H. Feng ${ }^{1,3}$, Y.L. Feng ${ }^{13}$, B. Gao ${ }^{1,3}$, C.D. Gao ${ }^{22}$, L.Q. Gao ${ }^{1,2,3}$, Q. Gao ${ }^{16}$, W. Gao ${ }^{22}$, M.M. Ge ${ }^{20}$, L.S. Geng ${ }^{1,3}$, G.H. Gong ${ }^{23}$, Q.B. Gou ${ }^{1,3}$, M.H. Gu ${ }^{1,3,6}$, F.L. Guo ${ }^{15}$, J.G. Guo ${ }^{1,2,3}$, X.L. Guo ${ }^{8}$, Y.Q. Guo ${ }^{1,3}$, Y.Y. Guo $^{1,2,3,13}$, Y.A. $\mathrm{Han}^{24}$, H.H. He $\mathrm{H}^{1,2,3}$, H.N. He ${ }^{13}$, J.C. He $\mathrm{H}^{1,2,3}$, S.L. He ${ }^{11}$, X.B. He ${ }^{18}$, Y. He ${ }^{8}$, M. Heller ${ }^{21}$, Y.K. $\mathrm{Hor}^{18}$, C. $\mathrm{Hou}^{1,3}$, H.B. $\mathrm{Hu}^{1,2,3}$, S. Hu${ }^{9}$, S.C. Hu${ }^{1,2,3}$, X.J. Hu${ }^{23}$, D.H. Huang ${ }^{8}$, Q.L. Huang ${ }^{1,3}$, W.H. Huang ${ }^{22}$, X.T. Huang $^{22}$, X.Y. Huang ${ }^{13}$, Z.C. Huang ${ }^{8}$, F. Ji ${ }^{1,3}$, X.L. Ji ${ }^{1,3,6}$, H.Y. Jia ${ }^{8}$, K. Jiang ${ }^{6,7}$, Z.J. Jiang ${ }^{20}$, C. Jin ${ }^{1,2,3}$, T. $\mathrm{Ke}^{1,3}$, D. Kuleshov ${ }^{25}$, K. Levochkin ${ }^{25}$, B.B. Li ${ }^{14}$, Cheng Li ${ }^{6,7}$, Cong Li ${ }^{1,3}, \mathrm{~F} \mathrm{Li}^{1,3,6}$, H.B. Li ${ }^{1,3}$, H.C. Li ${ }^{1,3}$, H.Y.

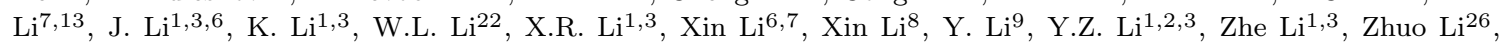

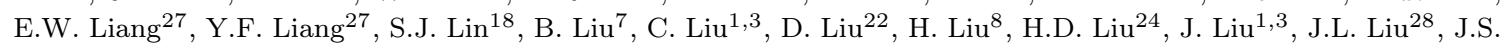

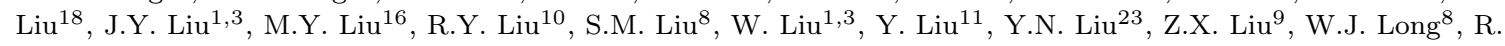

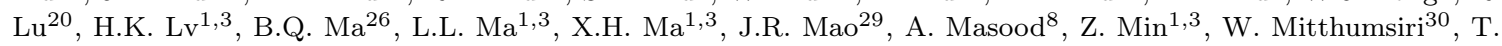
Montaruli $^{21}$, Y.C. $\mathrm{Nan}^{22}$, B.Y. Pang ${ }^{8}$, P. Pattarakijwanich ${ }^{30}$, Z.Y. Pei ${ }^{11}$, M.Y. Qi ${ }^{1,3}$, Y.Q. Qi ${ }^{14}$, B.Q. Qiao ${ }^{1,3}$, J.J. Qin $^{7}$, D. Ruffolo ${ }^{30}$, V. Rulev ${ }^{25}$, A. Sáiz ${ }^{30}$, L. Shao ${ }^{14}$, O. Shchegolev ${ }^{25,31}$, X.D. Sheng ${ }^{1,3}$, J.Y. Shi ${ }^{1,3}$, H.C. Song ${ }^{26}$, Yu.V. Stenkin ${ }^{25,31}$, V. Stepanov ${ }^{25}$, Y. Su ${ }^{32}$, Q.N. Sun ${ }^{8}$, X.N. Sun ${ }^{27}$, Z.B. Sun ${ }^{33}$, P.H.T. Tam ${ }^{18}$, Z.B. Tang ${ }^{6,7}$, W.W. Tian ${ }^{2,17}$, B.D. Wang ${ }^{1,3}$, C. Wang ${ }^{33}$, H. Wang ${ }^{8}$, H.G. Wang ${ }^{11}$, J.C. Wang ${ }^{29}$, J.S. Wang ${ }^{28}$, L.P. Wang ${ }^{22}$, L.Y. Wang 1,3 , R.N. Wang ${ }^{8}$, W. Wang ${ }^{18}$, W. Wang ${ }^{12}$, X.G. Wang ${ }^{27}$, X.J. Wang ${ }^{1,3}$, X.Y. Wang ${ }^{10}$, Y. Wang ${ }^{8}$, Y.D. Wang $^{1,3}$, Y.J. Wang ${ }^{1,3}$, Y.P. Wang ${ }^{1,2,3}$, Z.H. Wang ${ }^{9}$, Z.X. Wang ${ }^{20}$, Zhen Wang ${ }^{28}$, Zheng Wang ${ }^{1,3,6}$, D.M. Wei ${ }^{13}$, J.J. Wei ${ }^{13}$, Y.J. Wei ${ }^{1,2,3}$, T. Wen ${ }^{20}$, C.Y. Wu ${ }^{1,3}$, H.R. Wu ${ }^{1,3}$, S. $\mathrm{Wu}^{1,3}$, W.X. Wu $\mathrm{Wu}^{8}$, X.F. Wu ${ }^{13}$, S.Q. Xi ${ }^{1,3}, J^{\prime}$. $\mathrm{Xia}^{7,13}$, J.J. Xia ${ }^{8}$, G.M. Xiang ${ }^{2,15}$, D.X. Xiao ${ }^{16}$, G. Xiao ${ }^{1,3}$, H.B. Xiao ${ }^{11}$, G.G. Xin ${ }^{12}$, Y.L. Xin ${ }^{8}$, Y. Xing ${ }^{15}$, D.L. $\mathrm{Xu}^{28}$, R.X. Xu ${ }^{26}$, L. Xue ${ }^{22}$, D.H. Yan ${ }^{29}$, J.Z. Yan $^{13}$, C.W. Yang ${ }^{9}$, F.F. Yang ${ }^{1,3,6}$, J.Y. Yang ${ }^{18}$, L.L. Yang ${ }^{18}$, M.J.

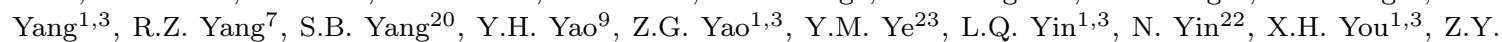
You $^{1,2,3}$, Y.H. Yu ${ }^{22}$, Q. Yuan ${ }^{13}$, H.D. Zeng ${ }^{13}$, T.X. Zeng ${ }^{1,3,6}$, W. Zeng ${ }^{20}$, Z.K. Zeng ${ }^{1,2,3}$, M. Zha ${ }^{1,3}$, X.X. Zhai ${ }^{1,3}$, B.B. Zhang ${ }^{10}$, H.M. Zhang ${ }^{10}$, H.Y. Zhang ${ }^{22}$, J.L. Zhang ${ }^{17}$, J.W. Zhang ${ }^{9}$, L.X. Zhang ${ }^{11}$, Li Zhang ${ }^{20}$, Lu Zhang ${ }^{14}$, P.F. Zhang ${ }^{20}$, P.P. Zhang ${ }^{14}$, R. Zhang ${ }^{7,13}$, S.R. Zhang ${ }^{14}$, S.S. Zhang ${ }^{1,3}$, X. Zhang ${ }^{10}$, X.P. Zhang ${ }^{1,3}$, Y.F. Zhang ${ }^{8}$, Y.L. Zhang ${ }^{1,3}$, Yi Zhang ${ }^{1,13}$, Yong Zhang ${ }^{1,3}$, B. Zhao ${ }^{8}$, J. Zhao ${ }^{1,3}$, L. Zhao ${ }^{6,7}$, L.Z. Zhao ${ }^{14}$, S.P. Zhao ${ }^{13,22}$, F. Zheng $^{33}$, Y. Zheng ${ }^{8}$, B. Zhou ${ }^{1,3}$, H. Zhou ${ }^{28}$, J.N. Zhou ${ }^{15}$, P. Zhou ${ }^{10}$, R. Zhou ${ }^{9}$, X.X. Zhou ${ }^{8}$, C.G. Zhu ${ }^{22}$, F.R. Zhu ${ }^{8}$, H. Zhu ${ }^{17}$, K.J. Zhu ${ }^{1,2,3,6}$ and X. Zuo ${ }^{1,3}$

${ }^{1}$ Key Laboratory of Particle Astrophyics \& Experimental Physics Division \& Computing Center, Institute of High Energy Physics, Chinese Academy of Sciences, 100049 Beijing, China.

${ }^{2}$ University of Chinese Academy of Sciences, 100049 Beijing, China.

${ }^{3}$ TIANFU Cosmic Ray Research Center, Chengdu, Sichuan, China.

${ }^{4}$ Dublin Institute for Advanced Studies, 31 Fitzwilliam Place, 2 Dublin, Ireland.

${ }^{5}$ Max-Planck-Institut for Nuclear Physics, P.O. Box 103980, 69029 Heidelberg, Germany.

${ }^{6}$ State Key Laboratory of Particle Detection and Electronics, China.

${ }^{7}$ University of Science and Technology of China, 230026 Hefei, Anhui, China.

${ }^{8}$ School of Physical Science and Technology \& School of Information Science and Technology, Southwest Jiaotong University, 610031 Chengdu, Sichuan, China.

${ }^{9}$ College of Physics, Sichuan University, 610065 Chengdu, Sichuan, China.

${ }^{10}$ School of Astronomy and Space Science, Nanjing University, 210023 Nanjing, Jiangsu, China.

${ }^{11}$ Center for Astrophysics, Guangzhou University, 510006 Guangzhou, Guangdong, China.

${ }^{12}$ School of Physics and Technology, Wuhan University, 430072 Wuhan, Hubei, China.

${ }^{13}$ Key Laboratory of Dark Matter and Space Astronomy, Purple Mountain Observatory, Chinese Academy of Sciences, 210023 Nanjing, Jiangsu, China.

${ }^{14}$ Hebei Normal University, 050024 Shijiazhuang, Hebei, China.

${ }^{15}$ Key Laboratory for Research in Galaxies and Cosmology, Shanghai Astronomical Observatory, Chinese Academy of Sciences, 200030 Shanghai, China.

${ }^{16}$ Key Laboratory of Cosmic Rays (Tibet University), Ministry of Education, 850000 Lhasa, Tibet, China.

${ }^{17}$ National Astronomical Observatories, Chinese Academy of Sciences, 100101 Beijing, China.

${ }^{18}$ School of Physics and Astronomy \& School of Physics (Guangzhou), Sun Yat-sen University, 519000 Zhuhai, Guangdong, China.

${ }^{19}$ Dipartimento di Fisica dell'Università di Napoli 'Federico II', Complesso Universitario di Monte Sant'Angelo, via Cinthia, 80126 Napoli, Italy.

${ }^{20}$ School of Physics and Astronomy, Yunnan University, 650091 Kunming, Yunnan, China.

${ }^{21}$ Departement de Physique Nuclaire et Corpusculaire, Facultde Sciences, Universit de Gen'eve, 24 Quai Ernest 
Ansermet, 1211 Geneva, Switzerland.

${ }^{22}$ Institute of Frontier and Interdisciplinary Science, Shandong University, 266237 Qingdao, Shandong, China.

${ }^{23}$ Department of Engineering Physics, Tsinghua University, 100084 Beijing, China.

${ }^{24}$ School of Physics and Microelectronics, Zhengzhou University, 450001 Zhengzhou, Henan, China.

${ }^{25}$ Institute for Nuclear Research of Russian Academy of Sciences, 117312 Moscow, Russia.

${ }^{26}$ School of Physics, Peking University, 100871 Beijing, China.

${ }^{27}$ School of Physical Science and Technology, Guangxi University, 530004 Nanning, Guangxi, China.

${ }^{28}$ Tsung-Dao Lee Institute \& School of Physics and Astronomy, Shanghai Jiao Tong University, 200240 Shanghai, China.

${ }^{29}$ Yunnan Observatories, Chinese Academy of Sciences, 650216 Kunming, Yunnan, China.

${ }^{30}$ Department of Physics, Faculty of Science, Mahidol University, 10400 Bangkok, Thailand.

${ }^{31}$ Moscow Institute of Physics and Technology, 141700 Moscow, Russia.

${ }^{32}$ Key Laboratory of Radio Astronomy, Purple Mountain Observatory, Chinese Academy of Sciences, 210023 Nanjing, Jiangsu, China.

${ }^{33}$ National Space Science Center, Chinese Academy of Sciences, 100190 Beijing, China. 